\title{
HepaRG-Progenitor Cell Derived Hepatocytes Cultured in Bioartificial Livers Are Protected from Healthy- and Acute Liver Failure-Plasma Induced Toxicity
}

\author{
Martien van Wenuma,b Philipp Treskes ${ }^{\mathrm{c}} \quad$ Aziza A.A. Adam ${ }^{a}$ \\ Vincent. A van der Mark ${ }^{b} \quad$ Aldo Jongejan $^{d} \quad$ Perry D. Moerland \\ Thomas M. van Gulik ${ }^{b}$ Ronald P.J. Oude Elferink ${ }^{a} \quad$ Robert A.F.M. Chamuleau $^{a}$ \\ Ruurdtje Hoekstraa,b \\ aTytgat Institute for Liver and Intestinal Research, Academic Medical Center (AMC), Amsterdam,

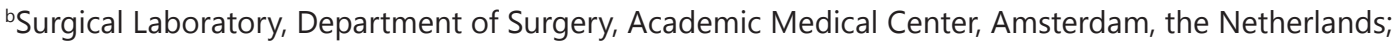 \\ 'Hepatology Laboratory, University of Edinburgh, Chancellor's Building, Royal Infirmary of Edinburgh, \\ Edinburgh, UK; ${ }^{d}$ Bioinformatics Laboratory, Department of Clinical Epidemiology, Biostatistics and \\ Bioinformatics, Amsterdam Public Health research institute, Academic Medical Center, Amsterdam, the \\ Netherlands
}

\section{Key Words}

Liver failure • Bioartificial liver $•$ Dedifferentiation $・ 3 D$ culture $\bullet$ Plasma toxicity $•$ HepaRG

\begin{abstract}
Background/Aims: For applicability of cell-based therapies aimed at the treatment of liver failure, such as bioartificial livers (BALs) and hepatocyte transplantation, it is essential that the applied hepatocytes tolerate exposure to the patient plasma. However, plasma from both healthy donors and acute liver failure (ALF) patients is detrimental to hepatocytes and hepatic cell lines, such as HepaRG. We aimed to elucidate the underlying mechanisms of plasmainduced toxicity against HepaRG cells in order to ultimately develop methods to reduce this toxicity and render HepaRG-BAL treatment more effective. Methods: Differentiated HepaRG cells cultured in monolayers and laboratory-scale BALs were exposed to culture medium, healthy human plasma, healthy porcine plasma and ALF porcine plasma. Healthy human plasma was fractionated based on size- and polarity, albumin depleted and heat treated to characterize the toxic fraction. The cells were assessed for viability by total protein content and trypan blue staining. Their hepatic differentiation was assessed on transcript level through qRT-PCR and microarray analysis, and on functional level for Cytochrome P450 3A4 activity and ammonia elimination. Mitochondrial damage was assessed by JC-1 staining and mitochondrial gene transcription. Results: Sixteen hours of healthy human plasma exposure did not affect viability, however, hepatic gene-transcript levels decreased dramatically and dose-dependently within
\end{abstract}


four hours of exposure. These changes were associated with early NF-kB signaling and a shift from mitochondrial energy metabolism towards glycolysis. Healthy human plasma-toxicity was associated with the dose-dependent presence of heat-resistant, albumin-bound and (partly) hydrophobic toxic compound(s). HepaRG cells cultured in BALs were partially protected from plasma-toxicity, which was mainly attributable to medium perfusion and/or 3D configuration applied during BAL culturing. The detrimental human plasma effects were reversible in BALcultured cells. Porcine ALF-plasma elicited mitotoxicity additional to the basal detrimental effect of porcine healthy plasma, which were only partially reversible. Conclusion: A specific fraction of human plasma reduces hepatic differentiation of HepaRG cultures, in association with early NF-KB activation. In addition, ALF-plasma elicits mitotoxic effects. These findings allow for a targeted approach in preventing plasma-induced cell damage.

(C) 2018 The Author(s)

Published by S. Karger AG, Basel

\section{Introduction}

Acute liver failure (ALF) and acute-on-chronic liver failure are syndromes associated with substantial mortality, for which there is an unmet need of therapeutic options [1], as orthotopic liver transplantation is limited by donor organ shortage. Two types of cell-based treatment modalities are under development: Bioartificial Liver (BAL) therapy [2-4] and hepatocyte transplantation $[5,6]$.

The performance of the applied cells in the presence of human plasma is of paramount importance, especially in BALs, that are designed as extracorporeal hepatocyte-bioreactors perfused with patient plasma through plasmapheresis. Healthy-donor human plasma ( $\mathrm{h}$ plasma) is known to induce intracellular lipid accumulation, stress and a decrease in hepatic functionality of primary hepatocytes and hepatic cell lines, through unclarified mechanisms [7, 8]. In addition, plasma from liver failure patients contains not only detrimental compounds which are normally detoxified by the liver, such as ammonia, bile acids, and lactate, but also compounds associated with inflammation and infection, such as endogenous damage-associated molecular patterns released from necrotic cells, cytokines and chemokines, as well as lipopolysaccharides and other stimulators of innate immune response due to bacterial translocation [9-11].

The AMC-BAL is a bioreactor that holds liver cells in 3D-configuration, in a spirally wound, non-woven matrix, interwoven with capillaries which supply oxygen-enriched gas $[12,13]$. Liver cells come in direct contact with patient plasma during treatment. Currently, the device is loaded with the human liver progenitor cell line HepaRG [14, 15], and was proven efficacious in prolonging survival time of rats with ischemic ALF [4]. Previously, we described that healthy- and ALF-rat plasma induced toxicity in HepaRG cells cultured in monolayers and in BALs $[16,17]$.

In this study we show that plasma has a rapid detrimental effect on hepatic differentiation and functionality of HepaRG cells. In order to develop protective strategies, we studied the underlying mechanism of plasma-induced toxicity and the toxic fraction of ${ }_{\text {h }}$ plasma. We further studied whether culture procedures or different culture platforms could inhibit or reverse the plasma-induced toxicity. Finally, we assessed whether ALF plasma induced additional toxicity compared to plasma of healthy subjects, using pig plasma.

\section{Materials and Methods}

\section{Cell culture and plasma exposure procedures}

HepaRG cells (Biopredic International) were cultured in supplemented Williams' E medium without dimethyl sulfoxide (DMSO), with 10\% fetal bovine serum, as described [14, 18]. Differentiated monolayers were acquired after seeding 1:5 in 12-well plates and culturing for 28 days under control conditions, i.e. statically under an atmosphere of $5 \% \mathrm{CO}_{2}, 75 \% \mathrm{~N}_{2}$ and $20 \%$ of $\mathrm{O}_{2}$. Monolayers cultured under hyperoxia were kept under normoxia until reaching confluence at day 14 and then transferred to $5 \% \mathrm{CO}_{2}, 55 \% \mathrm{~N}_{2}$ and 


\section{Cellular Physiology Cell Physiol Biochem 2018;48:2189-2204 \begin{tabular}{l|l|l}
\hline DOI: 10.1159/000492560 & $\begin{array}{l}\text { () 2018 The Author(s). Published by S. Karger AG, Basel } \\
\text { www.karger.com/cpb }\end{array}$
\end{tabular}}

$40 \%$ of $\mathrm{O}_{2}$ until day 28.

3D 'Bal-in-a-dish' (Baliad) cultures were seeded into $28.2 \mathrm{~mm}^{2}$ pieces of non-woven matrix and kept in medium in 12-well plates under control conditions until day fourteen, after which they were transferred to an orbital-shaker incubator under normoxia and shaken at $60 \mathrm{rpm}$ until day 28.

Laboratory-scale BALs ( 9 mL volume) were loaded with $0.6 \mathrm{~g}$ cryopreserved HepaRG cells and cultured for 21 days as described previously [19] before commencing plasma-exposure-experiments.

To test the effect of plasma exposure, culture medium was replaced by undiluted plasma, except for the experiment with serial dilutions, for which plasma was diluted with fresh culture medium as indicated. As a negative control, cells were kept in fresh culture medium. Plasma exposure times varied between experiments, as indicated.

To test whether HepaRG-BAL cultures could recover from plasma-toxicity, plasma was removed from the BALs after 16h exposure by a single-pass flush with fresh culture-medium, after which the system was filled entirely with fresh culture medium and allowed to recover for 24 hours. Matrix samples for RNA isolation were obtained before plasma exposure (control) and immediately after plasma exposure, as described previously [20].

\section{Plasma}

Healthy human plasma $\left({ }_{\mathrm{h}}\right.$ plasma) derived from mixed-gender pooled donors (Sera Laboratories International Ltd.). Healthy porcine plasma $\left({ }_{p}\right.$ plasma) derived from landrace pigs. For porcine ALF plasma ( ${ }_{\mathrm{pALF}}$ plasma), ALF was induced as described [21] in a $31 \mathrm{~kg}$ female landrace pig by intravenous administration of paracetamol $1.5 \mathrm{~g} / \mathrm{kg}$ body weight over 12 hours. After 21 hours the animal went into cardiovascular failure, and subsequently plasma was acquired by exsanguination. Liver failure was confirmed by biochemical analysis (Table 1). All procedures involving animals were conducted in agreement with the Animals (Scientific Procedures) Act 1986 under (UK Home Office) Project License 60/4557, and after approval by the Roslin Institute's Animal Welfare and Ethical Review Board.

All plasmas were anticoagulated with Lithium-Heparin, filter-sterilized and supplemented with penicillin $(100 \mathrm{U} / \mathrm{mL})$ and streptomycin $(100 \mu \mathrm{g} / \mathrm{mL})$ before exposure to HepaRG cells. Standard HepaRG medium contains the equivalent concentrations of penicillin and streptomycin.

\section{Plasma fractionation or treatment}

To characterize the toxic component in ${ }_{h}$ plasma, we fractioned or treated this plasma with different techniques. Fractionation by size was performed by filtering with $100 \mathrm{kDa}$ Molecular weight cut-off filters (Amicon Ultra-4, Merck), which are specified to retain molecules of over $100 \mathrm{kDa}$, including albumin, which we confirmed.

Fractionation by polarity was performed by Bligh and Dyer extraction [22], after which the hydrophobic fraction was dried under a stream of nitrogen, resuspended and sonicated in DMSO, which was then diluted in culture medium to equal volume as the original plasma sample (final concentration of DMSO was 1\%). Vehicle control treatment was performed with culture medium that was treated the same way $(n=6,2$ independent experiments).

Albumin depletion was done by concentrating $3 \mathrm{~mL}$ of plasma with $100 \mathrm{kDa}$ Molecular weight cut-off filters until $40 \mathrm{uL}$ retentate, which was then resuspended in a $20 \mathrm{mM}$ sodium phosphate binding-buffer (pH 7.0), as recommended by manufacturer, and exposed to $7 \mathrm{~mL}$ albumin-binding Blue Sepharose 6 Fast Flow beats (GE Healthcare) for 3 hours at $4^{\circ} \mathrm{C}$ and $30 \mathrm{~min}$ at room temperature on an orbital shaker at 100 rpm. Subsequently, the mix was concentrated over $100 \mathrm{kDa}$ Molecular weight cut-off filters and the retentate was diluted in culture medium to a final volume of $3 \mathrm{~mL}$. Control plasma was treated with the same protocol, except for the exposure to Blue Sepharose beats ( $n=6,2$ independent experiments). Albumin was under the level of detection in the albumindepleted plasma, as confirmed by the clinical chemistry laboratory (data not shown).

Heat treatment of $100 \%$ plasma consisted of a 45 -minutes exposure to $58^{\circ} \mathrm{C}$ in a block heater ( $n=6,2$ independent experiments).

Table 1. Biochemical profile of ${ }_{\mathrm{pALF}}$ plasma. Baseline value is before paracetamol administration. AST=Aspartate Aminotransferase; Mir-122 = MicroRNA-122; FV= clotting factor five activity; FIII=clotting factor eight activity.

\begin{tabular}{lcc}
\hline & Baseline & Time of death \\
\hline AST(U/L) & 37 & 243 \\
Ammonia (uM) & 58 & 316 \\
Mir-122 (Fold change vs baseline) & 1 & 160 \\
FV/FVIII (ratio) & 1.07 & 0.13 \\
\hline
\end{tabular}




\section{Cellular Physiology Cell Physiol Biochem 2018;48:2189-2204

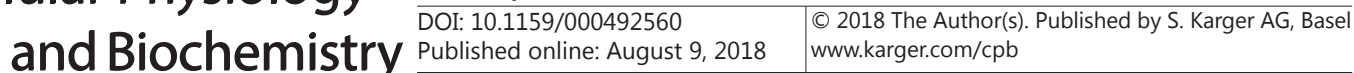

van Wenum et al: Healthy- and Acute Liver Failure -Plasma Induced Toxicity

Cell viability assessment

HepaRG monolayer cultures were exposed to ${ }_{h}$ plasma or culture medium for 16 hours, washed $2 \mathrm{x}$ with PBS and then assessed for toxicity by two methods ( $n=4,2$ independent experiments). Firstly the cultures were incubated with trypan blue $0.4 \%$ solution (Sigma) diluted 1:4, after which the cultures were immediately assessed for viability by microscopy. Secondly, the total protein content/well was determined as described [18].

\section{Function tests}

Hepatic function tests were performed as previously described [4]. Briefly, test medium was prepared from HepaRG medium supplemented with $\mathrm{NH}_{4} \mathrm{Cl}(1.5 \mathrm{mM})$, L-lactate $(2.5 \mathrm{mM})$, D-galactose $(2.27 \mathrm{mM})$ and testosterone $(125 \mu \mathrm{M})$. Monolayers and BALs were exposed to 1.5 and $110 \mathrm{~mL}$ of test medium respectively and samples taken after 0,6 and 24 hours for monolayers and 0, 30, 60, 120, 240, 480 and 1440 min for BALs. Concentrations of ammonia were quantified using the Megazyme Ammonia Assay kit (Megazyme International). Cytochrome P450 3A4 (CYP3A4) activity was measured in BALs by the quantification of 6 $\beta$-hydroxytestosterone as described [4], and in monolayers using the P450-Glo ${ }^{\mathrm{TM}} \mathrm{CYP} 3 \mathrm{~A} 4$ with LuciferinIPA assay according to the manufacturer's instruction (Promega). All metabolic rates were normalized to total protein as described [18]. Synthetic properties could not be assessed adequately, due to some retention of plasma proteins after plasma exposure.

\section{RNA isolation, qRT-PCR and microarray analysis}

Cells in monolayers or representative samples of BAL-matrix were lysed in $600 \mu \mathrm{L}$ RLT buffer (RNeasy minikit, Qiagen) from which RNA was isolated according to manufacturer's instructions. Transcript levels were determined by qRT-PCR using gene-specific reverse transcriptase (RT)-primers and touchdown qPCR protocol and normalized to $18 \mathrm{~S}$ ribosomal RNA, as previously described [23]. Where expressed as \% of control, the data were normalized to the average of non-treated control cells within each independent experiment. For the microarray experiment RNA was isolated after $0,1,2,4$ or 8 hours exposure to ${ }_{\mathrm{h}}$ plasma ( $\mathrm{n}=3$ independent $\sim 1 \mathrm{~cm}^{2}$ monolayer cultures per group) RNA was biotinylated with the cRNA labeling kit (Ambion) and hybridized to Illumina HumanHT-12 v4 arrays (Illumina) after randomization of the samples. Scanning was performed on the Illumina iScan (Illumina). Image analysis and extraction of raw expression data was performed with Illumina GenomeStudio v2011.1 Gene Expression software with default settings (no background subtraction and no normalization). The microarray data were analyzed with Bioconductor packages (version 3.0) using the statistical software environment R (version 3.1.3). Raw data normalization was performed on the Illumina sample and control probe profiles by normexp-by-control background correction, quantile normalization, and log2 transformation using the limma package (version 3.22.7). The arrayQualityMetrics package (version 3.22.1) was used to assess that the microarray data was of good quality. Probes with a detection P-value $>0.05$ (non-expressed) on all arrays (16, 560 of 47, 323 probes) were filtered out. Differential expression between time points was assessed using a moderated t-test using the linear model framework from the limma package.

Resulting P-values were corrected for multiple testing using the Benjamini-Hochberg false discovery rate. Corrected P-values $\leq 0.05$ were considered statistically significant. Probes were reannotated using the IlluminaHumanv4.db package (version 1.24.0). Gene sets from the hallmark collection and two liver-specific gene sets from the C2 collection, HSIAO_LIVER_SPECIFIC_GENES and SU_LIVER [24, 25], were retrieved from the Molecular Signatures Database (MSigDB) v5.1 (Entrez Gene ID version). Gene set enrichment analysis was performed using CAMERA (limma package) and gene set variation analysis (GSVA) was performed using the GSVA package (version 1.14.1). Sample-specific gene set enrichment scores calculated by GSVA were clustered using Pearson correlation as distance measure and complete linkage as agglomeration method (function hclust). Top-10 upregulated genes were cross-referenced against the Boston University Gilmore Lab NF- $\mathrm{kB}$ target gene set (http://www.bu.edu/nf-kb/gene-resources/target-genes/).

\section{Mitochondrial membrane potential and mitochondrial abundance}

Mitochondrial membrane potential was determined using JC-1 staining. This cationic dye emits green fluorescence in the cytosol of the cells (monomeric form) and red fluorescence when aggregates are formed (dimeric form) in active mitochondrial membrane [26]. BAL matrix samples ( $6 \mathrm{~mm} \times 6 \mathrm{~mm}$ ) were incubated 


\section{Cellular Physiology Cell Physiol Biochem 2018;48:2189-2204 \\ and Biochemistry Published onnIne: August 9, $2018 \quad \begin{aligned} & \text { DO 2018 The Author(s). Published by S. Karger AG, Basel } \\ & \text { www.karger.com/cpb }\end{aligned}$ \\ van Wenum et al: Healthy- and Acute Liver Failure -Plasma Induced Toxicity}

with $0.5 \mathrm{~mL} 4 \mu \mathrm{M} \mathrm{JC}-1$ (Invitrogen) for $30 \mathrm{~min}$ at $37^{\circ} \mathrm{C}$. Simultaneously, the cells were incubated with $1 \mathrm{uM}$ verapamil (Sigma) to inhibit the efflux of JC-1 through the activity of ATP binding cassette subfamily B member 1. Red to green fluorescence (F $2585 / F \lambda 510)$ was quantified on a NOVOstar microplate reader (BMG Labtech).

The ratio of mitochondrial DNA (mtDNA) to nuclear DNA (nucDNA) was assessed as a measure of mitochondrial abundance expressed as the weighted mean. Total DNA was isolated from AMC-BAL matrix samples (6 mm x $6 \mathrm{~mm}$ ) using the QIAamp DNA kit (QIAGEN) according to the manufacturer's specifications. 2 nuclear encoded genes, i.e. CCAAT/enhancer binding protein alpha (CEBPa) and $\mathrm{N}$-acetyl transferase (NAT), and 2 mitochondrial-encoded genes, i.e. mitochondrial-NADH dehydrogenase subunit1 (MT-ND1) and mitochondrial cytochrome c oxidase subunit 3 (MT-CO3) were quantified by qPCR.

\section{Statistical analyses}

Data were analyzed and processed for graphical representation in Prism 7.01 (GraphPad). Values are expressed as mean \pm standard deviation (SD). Student's t-tests were used when comparing two groups and 1-way ANOVA when comparing multiple groups. All results were corrected for multiple testing according to the Holm-Sidak method. Significance was reached if $P<0.05$. The data produced in BALs were $n=3,3$ independent experiments, compared to monolayer cultures $n=8,3$ independent experiments. Plasmatreatment experiments were performed as indicated in text.

\section{Results}

Healthy-donor human plasma has a rapid, dose-dependent detrimental effect on the hepatic differentiation of HepaRG-derived hepatocytes

We determined the effects of ${ }_{\mathrm{h}}$ plasma exposure on HepaRG monolayer cultures on total protein content, morphology, ammonia elimination rate, CYP3A4 activity and the transcript levels of the hepatic genes CYP3A4, hepatic nuclear factor 4 Alpha (HNF4A) and arginase 1 (ARG1), which were previously established to be highly responsive to plasma exposure $[16,17]$. HepaRG monolayers showed a rapid decrease in transcript levels of these hepatic genes when exposed to $100 \%$ plasma (Fig. 1A). After 4 hours exposure, HNF4A transcript level was decreased to $17 \pm 2 \%$ of the level in control cultures, while CYP3A4 and $A R G 1$ transcript levels decreased more gradually to $5 \pm 2 \%$ and $7 \pm 2 \%$ respectively after 24 hours. Decrease in transcript levels of these genes was accompanied by profound morphological changes. The clusters of hepatocyte-like polygonal cells in HepaRG cultures disappeared (Fig. 1B) and cell-cell contact was lost after 16 hours exposure to ${ }_{\mathrm{h}}$ plasma. There was no significant cell death as determined by total protein content (Fig. 1C) or trypan blue staining (Fig. 1D). However, rates of ammonia elimination and CYP3A4 activity had decreased to $48 \pm 21 \%$ and $29 \pm 7 \%$ of levels in control cultures, respectively (Fig. 1E). A 16 hours exposure of monolayers to ${ }_{h}$ plasma in escalating concentrations revealed a plasma-concentration-dependent decrease of hepatic gene transcript levels of CYP3A4, HNF4 and ARG1 (Fig. 1F). The transcript levels of the control ( $0 \%$ plasma-exposure) group were significantly higher $(P<0.05)$ compared to all other groups, with the exception of ARG1 versus $25 \%$ plasma. The gene transcript levels were also significantly lower after exposure to $100 \%$ compared to $25 \%$ plasma for all three genes $(P<0.05)$.

Together, these data indicate that ${ }_{\mathrm{h}}$ plasma has a rapid and dose-dependent toxic effect on the differentiation of HepaRG monolayers, although their viability is still unaffected.

\section{Detrimental plasma fraction is hydrophobic, albumin-bound, and heat-stabile}

Next, we determined whether the detrimental effect of ${ }_{h}$ plasma was due to a lack of indispensable culture medium components or to the presence of a toxic fraction, by exposing monolayers to different fractions of ${ }_{\mathrm{h}}$ plasma for $16 \mathrm{~h}$ and testing the effect on transcript levels of ARG1, HNF4A, and CYP3A4. ${ }_{\mathrm{h}}$ Plasma filtered through $100 \mathrm{kDa}$ molecular weight cut-off filters (which also depleted albumin, Mw $67 \mathrm{kDa}$ ), did not negatively affect transcript levels 


\section{Cellular Physiology

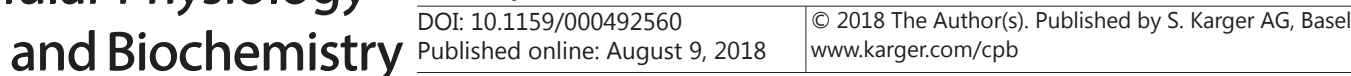 \\ van Wenum et al: Healthy- and Acute Liver Failure -Plasma Induced Toxicity}

Fig. 1. Effect of ${ }_{\mathrm{h}}$ plasma on differentiated HepaRG cells. A.) Transcript levels of the hepatic genes ARG1, CYP3A4 and HNF4A in HepaRG cells after $0.5,1,2,4,8,12$ and 24 hours of exposure to ${ }_{\mathrm{h}}$ plasma, expressed as a \% of the transcript levels in non-exposed cells. B.) Morphology of HepaRG cells before and after 16 hours exposure to ${ }_{\mathrm{h}}$ plasma, bar $=50 \mu \mathrm{m}$. C.) Total protein content per well after 16 hours exposure to ${ }_{h}$ plasma or culture medium. D.) Viability evaluated by trypan blue exclusion test of HepaRG cells exposed for 16 hours to culture medium control or ${ }_{\mathrm{h}}$ plasma (stained cells have lost membrane integrity and are not viable). Red arrows indicate trypan blue positive cells. E) Hepatic functions ammonia elimination and CYP3A4 activity before and after 16 hours exposure to ${ }_{h}$ plasma. F.) Hepatic gene transcript levels after 16 hours exposure to ${ }_{\mathrm{h}}$ plasma in different concentrations. $*=P<0.05$ versus control.

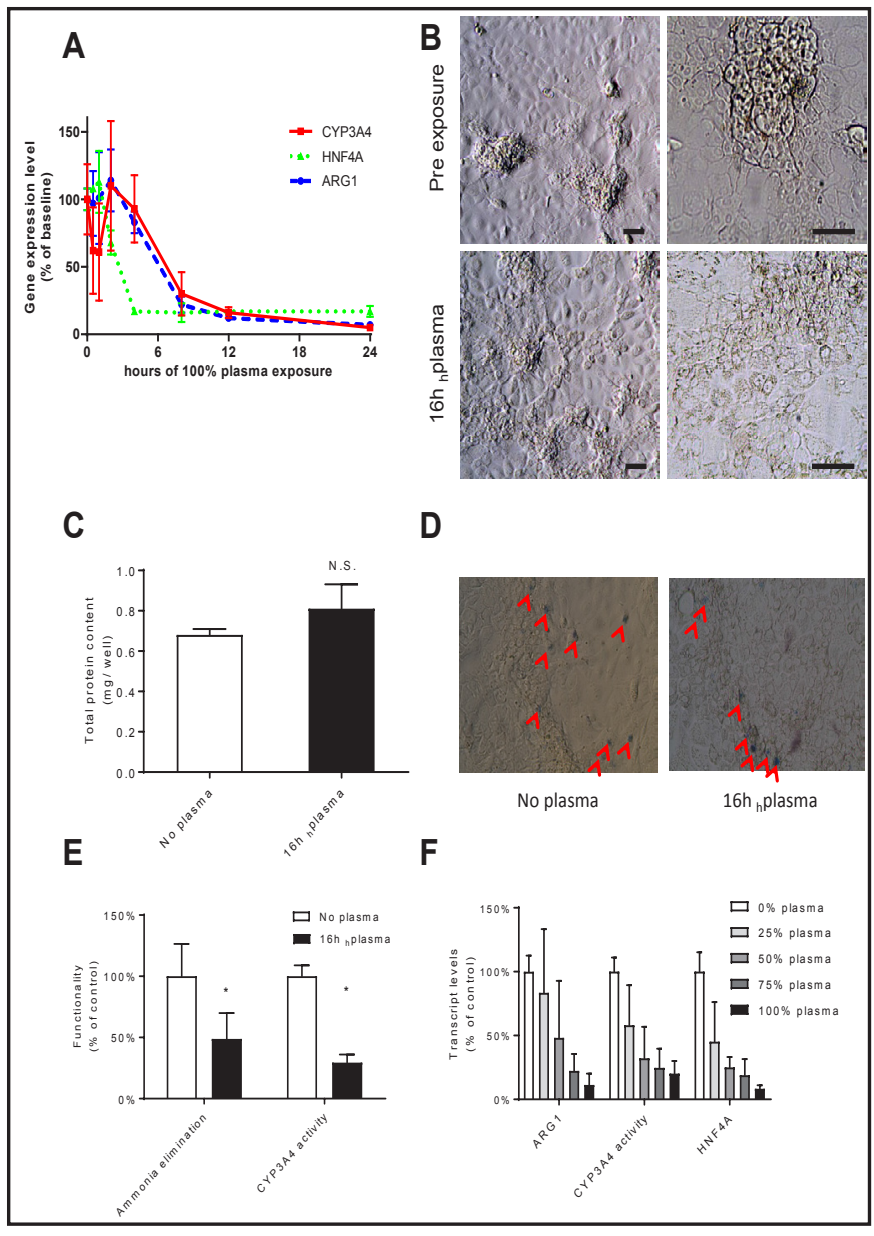

of $A R G 1$ or $H N F 4 A$, while $C Y P 3 A 4$ was even significantly upregulated compared to control cultures (Fig. 2A). This supported the presence of a toxic fraction rather than depletion of essential culture medium components by $100 \%$ plasma incubation.

${ }_{\mathrm{h}}$ Plasma that was albumin-depleted induced a significantly smaller decrease in transcript levels of ARG1 $(P<0.01)$ and HNF4A $(P<0.001)$ and an increase in CYP3A4 transcript levels $(P<0.001)$ compared to control-treated ${ }_{h}$ plasma (Fig. $\left.2 \mathrm{~B}\right)$. Heat treated ${ }_{h}$ plasma $\left(58^{\circ} \mathrm{C} 45 \mathrm{~min}\right)$ yielded similar effects as untreated plasma (Fig. 2C) and finally, the hydrophobic plasma fraction also reduced transcript levels of ARG1 and HNF4A, but not of CYP3A4 (Fig. 2D).

These data show that the cytotoxic effect of plasma is due to an albumin-bound, heatstabile fraction, that is, at least partly, hydrophobic, and not due to a lack of essential culture medium factors.

\section{Plasma-induced damage is associated with rapid activation of NF- $\kappa B$ target genes}

To investigate the pathways involved in plasma-induced toxicity, we compared the whole-genome transcriptomes of HepaRG monolayers exposed to ${ }_{h}$ plasma for different durations by microarray analysis. Since loss of hepatic gene transcription commenced within eight hours (Fig. 1A), we compared monolayers after 1, 2, 4 and 8 hours of ${ }_{\mathrm{h}}$ plasma exposure with control monolayers. There were profound and rapid changes in transcriptomes; after 1, 2, 4 and 8 hours there were 134, 1154, 5548 and 6584 genes differentially expressed, respectively, compared to control cultures (Fig. 3A).

Of the top-10 upregulated genes at the different time points versus control cultures (Table 2), 11 out of 26 unique genes were NF- $\kappa B$ targets (depicted in bold). Gene set enrichment analysis on the hallmark collection of the Molecular Signatures Database (MSigDB) v5.1 


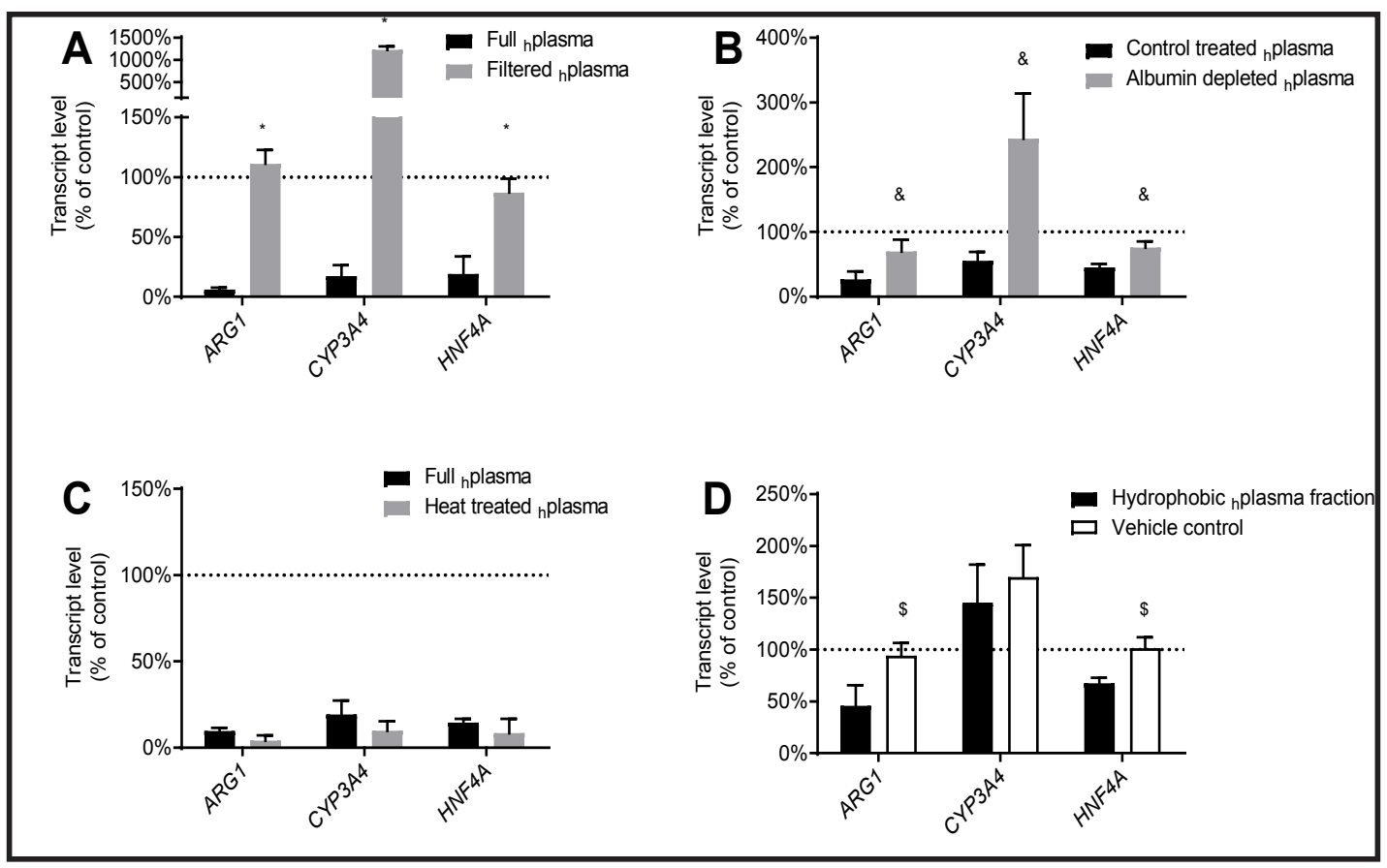

Fig. 2. Toxicity of ${ }_{\mathrm{h}}$ plasma fractions. Transcript levels of the hepatic genes ARG1, CYP3A4 and HNF4A in differentiated HepaRG monolayers exposed to different fractions of ${ }_{h}$ plasma for 16 hours, expressed as a \% of the transcript levels in non-exposed cells. A.) Plasma filtered through a molecular weight cut-off filter compared to untreated ${ }_{\mathrm{h}}$ plasma. B.) Albumin depleted plasma compared to control-treated plasma. C.) Heat treated ${ }_{h}$ plasma compared to untreated ${ }_{h}$ plasma. D.) The hydrophobic plasma fraction compared to treatment vehicle control. ${ }^{*}=P<0.05$ versus monolayers exposed to untreated ${ }_{\mathrm{h}}$ plasma. $\&=P<0.05$ versus monolayers exposed to control-treated ${ }_{\mathrm{h}}$ plasma. $\$=P<0.05$ versus monolayers exposed to vehicle-treated control.

showed that genes regulated by NF- $\mathrm{BB}$ in response to TNF were the most upregulated gene set after two hours exposure (Fig. 3B-C). Energy metabolism changed drastically, as oxidative phosphorylation was strongly downregulated at later time points. In line with the qRT-PCR data (Fig. 1A), enrichment analysis of two previously described gene sets of liver-specific genes (1: [25] and 2:[24]) showed a profound decrease of hepatic gene transcript levels at 4 and 8 hours after exposure (Fig. 3B-C). To confirm the activation of NF- $\kappa$ B, we quantified gene transcript levels of target genes interleukin 6 (IL6) and interleukin 8 (IL8) after two hours exposure to human plasma (Fig. 3D). Both genes were significantly upregulated in the plasma-exposed group (1715\% and $762 \%$ of control respectively).

\section{BAL-culturing protects against plasma-induced deterioration, which may be explained by} $3 D$ configuration and medium perfusion

HepaRG cultures in BALs were less sensitive to ${ }_{\mathrm{h}}$ plasma-induced damage compared to monolayers. After $16 \mathrm{~h}$ of exposure, ammonia elimination was significantly decreased in monolayer cultures, but not in BAL cultures (Fig. 4A), while CYP3A4 activity was significantly decreased in both cultures, although significantly less in BAL cultures than in monolayers (2.2-fold versus 3.4-fold reduction) (Fig. 4B). After exposure, transcript levels of ARG1, CYP3A4 and HNF4A were declined 6.9-, 5.4- and 4.0-fold, respectively, in monolayer cultures, while in BAL cultures $C Y P 3 A 4$ transcript levels were unchanged after plasma exposure and ARG1 and HNF4A transcript levels were less reduced, (2.2- and 1.8-fold) (Fig. 4C-E).

Three main differences between the monolayer and BAL culture systems are the level of oxygenation ( $20 \%$ vs $40 \%$ 02, respectively), the configuration (2D vs 3D, respectively) and medium perfusion (absent or present, respectively). To assess which of these factors 
Fig. 3. Differentially expressed genes and gene sets. A.) Venn diagram of differentially expressed (adjusted $P<0.05$ ) genes of the monolayer cultures exposed to ${ }_{\mathrm{h}}$ plasma for 1 to 8 hours compared to unexposed cultures. B.) Heatmap of the sample-specific gene set enrichment scores calculated by gene set variation analysis (GSVA) on the hallmark gene sets and two previously described human liverspecific gene sets. C.) A high detail full-resolution version of Fig. 3B. D) IL6 and IL8 transcript levels after 2 hour exposure to plasma or culture medium (control). EMT= Epithelial to mesenchymal transition. Ox. phosphorylation = Oxidative phosphorylation $*=P<0.05$ versus control.

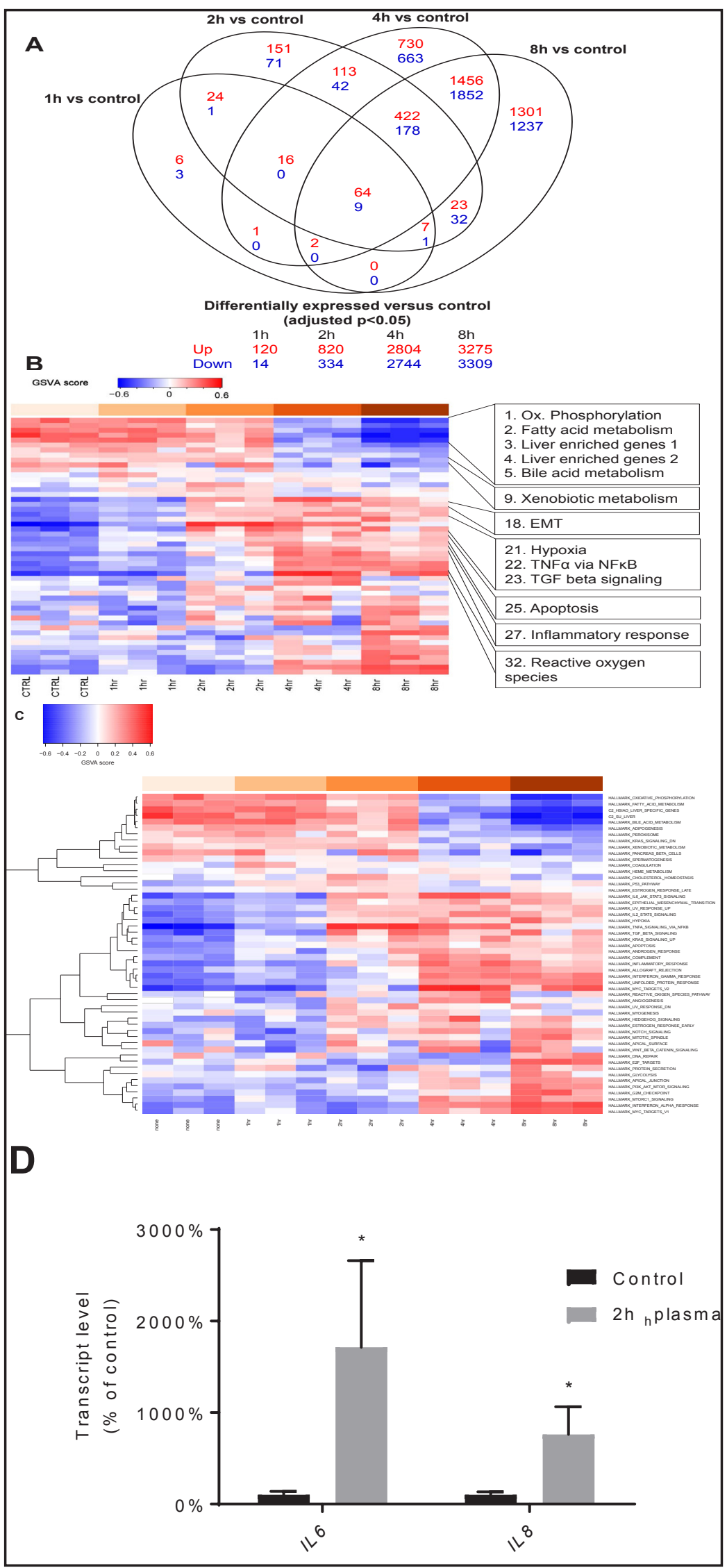


contribute to the relative resistance to ${ }_{h}$ plasma toxicity in BAL cultures versus monolayer cultures, we exposed medium-perfused 3D cultures under ambientnormoxia (Baliad cultures) and static monolayer cultures under $40 \%$ of ambient oxygen to ${ }_{\mathrm{h}}$ plasma and compared the decrease in transcript levels of ARG1, CYP3A4 and HNF4A between standard monolayer and BAL cultures. The effect of ${ }_{\mathrm{h}}$ plasma on transcript levels did not differ between normoxic or hyperoxic monolayers. In Baliad cultures, the transcript levels of $A R G 1$ and CYP3A4, but not of HNF4A, were less decreased by ${ }_{\mathrm{h}}$ plasma exposure compared to monolayer cultures. In addition the transcript levels of all three tested genes were not significantly different after ${ }_{\mathrm{h}}$ plasma exposure in BAL- and Baliad-cultures, indicating that medium perfusion and 3D culture may be important contributing factors to the protective effect of BAL-culturing.

Loss of hepatic gene
transcription is reversible in
BALs after exposure to healthy
human plasma
The previous experiments
man plasma, because of the
rcity of human ALF plasma.

Table 2. Top-10 upregulated genes after different durations of ${ }_{\mathrm{h}}$ plasma exposure compared to non-exposed controls. Values represent fold change versus control cultures. Genes written in bold are recognized targets of NF- $\mathrm{kB}$ according to the Boston university Gilmore Lab NF- $\kappa$ B target gene set.

\begin{tabular}{lcccc}
\hline & 1h exposure & 2h exposure & 4h exposure & 8h exposure \\
\hline CCL20 & $\mathbf{8 . 6}$ & $\mathbf{3 6 . 8}$ & $\mathbf{2 6 . 0}$ & $\mathbf{1 0 . 6}$ \\
CYR61 & 6.1 & & & \\
EDN2 & 8.6 & & & \\
EGR1 & $\mathbf{5 . 7}$ & & & \\
EGR2 & 9.8 & & & \\
FOS & $\mathbf{3 6 . 8}$ & & & \\
GDF15 & 6.5 & & & \\
IL6 & $\mathbf{8 . 0}$ & $\mathbf{4 8 . 5}$ & $\mathbf{1 8 . 4}$ & $\mathbf{1 1 . 3}$ \\
IL8 & $\mathbf{6 . 1}$ & $\mathbf{1 0 . 6}$ & & \\
KLF10 & $\mathbf{6 . 5}$ & & & \\
CCL2 & & $\mathbf{2 6 . 0}$ & & \\
CCL7 & & 10.6 & 22.6 & \\
MAFF & & 13.9 & & \\
SERPINB2 & & 18.4 & 48.5 & 42.2 \\
SOCS3 & & 12.1 & & \\
TRIB1 & & 11.3 & & \\
TUFT1 & & 11.3 & & \\
AKAP12 & & & 9.8 & \\
IGFBP1 & & & $\mathbf{3 4 . 3}$ & $\mathbf{2 4 . 3}$ \\
IL1RL1 & & & $\mathbf{1 6 . 0}$ & $\mathbf{8 . 6}$ \\
MMP3 & & & $\mathbf{2 1 . 1}$ & $\mathbf{1 0 . 6}$ \\
TGFB3 & & & 17.1 & \\
TNC & & & $\mathbf{9 . 8}$ & $\mathbf{1 0 . 6}$ \\
ANGPTL4 & & & & 11.3 \\
HMGA1 & & & & 8.6 \\
IGFBP3 & & & & 17.1 \\
\hline
\end{tabular}

Fig. 4. Effect of culture platform on ${ }_{\mathrm{h}}$ plasma toxicity. A-B.) Functionality of HepaRG monolayer or BALs cultures in culture medium (control) or after exposure to ${ }_{\mathrm{h}}$ plasma for $16 \mathrm{~h}$, expressed as a $\%$ of unexposed cultures in the same cultureplatform: A.) Ammonia elimination, B.) CYP3A4 activity. C-E.) Transcript levels of hepatic genes in HepaRG cells cultured in monolayers under normoxia or hyperoxia, in Baliads or in BALs, exposed to ${ }_{h}$ plasma for $16 \mathrm{~h}$, expressed as a \% of unexposed cultures in the same culture-platform: C) $A R G 1$, D) CYP3A4, E) HNF4A. $\#=P<0.05$ versus ${ }_{\mathrm{h}}$ plasma-exposed monolayer. ${ }^{*}=P<$ 0.05 versus monolayer normoxia. $\$=P<$ 0.05 versus unexposed cultures in the same culture platform.

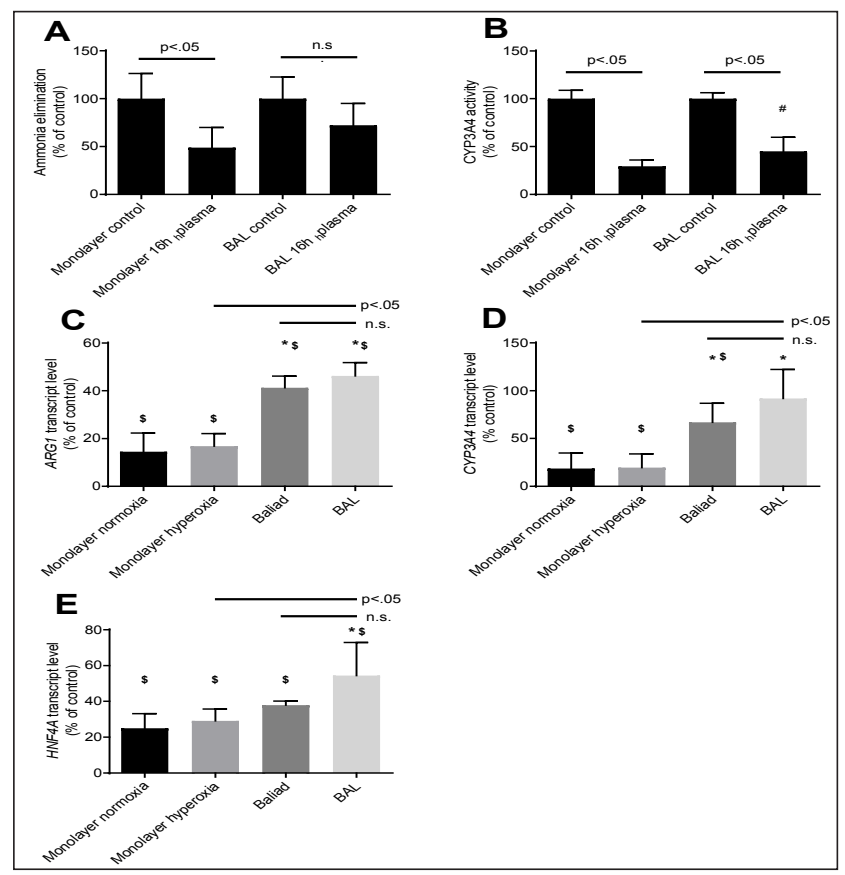


However, when applied clinically, the BAL-system will be exposed to plasma from patients suffering from ALF, which has a different composition from healthy plasma. The effects of the ALF contribution on plasma toxicity were therefore analyzed by investigating hepatic transcript levels of BALs exposed to plasma samples from: healthy pigs ( ${ }_{\mathrm{p}}$ plasma) and pigs with acetaminophen-induced ALF ( pALF $_{\text {p }}$ plasma). As a control for species differences, ${ }_{\mathrm{h}}$ plasmaexposed BALs were also included. After plasma exposure, all BALs were allowed to recover in culture medium for 24 hours and transcript levels were again measured to assess the reversibility of the plasma-induced toxicity.

HepaRG BALs exposed to ${ }_{\mathrm{h}}$ plasma, ${ }_{\mathrm{p}}$ plasma and ${ }_{\mathrm{pALF}}$ plasma all showed a significant decrease in transcript levels of $A R G 1$ and $H N F 4 A$ (Fig. 5), while only the latter two groups showed a decrease in CYP3A4 transcript levels.

The level of recovery from the plasma exposure varied with the origin of the plasma. After recovery from ${ }_{\mathrm{h}}$ plasma exposure, transcript levels of all three genes were higher compared to post-exposure levels, up to or exceeding control levels. After recovery from plasma exposure, only CYP3A4 transcript levels increased significantly, although HNF4A transcript levels no longer differed significantly from the pre-exposure levels. After recovery from ${ }_{\text {pALF }}$ plasma exposure, there was no significant upregulation of any of the three genes compared to post-exposure levels, although there was no significant difference anymore to pre-exposure level for HNF4A transcripts. Thus, we conclude that the decrease of gene transcript levels in BALs after 16 hours plasma exposure is partially reversible, and the degree of reversibility varies with the origin of plasma.

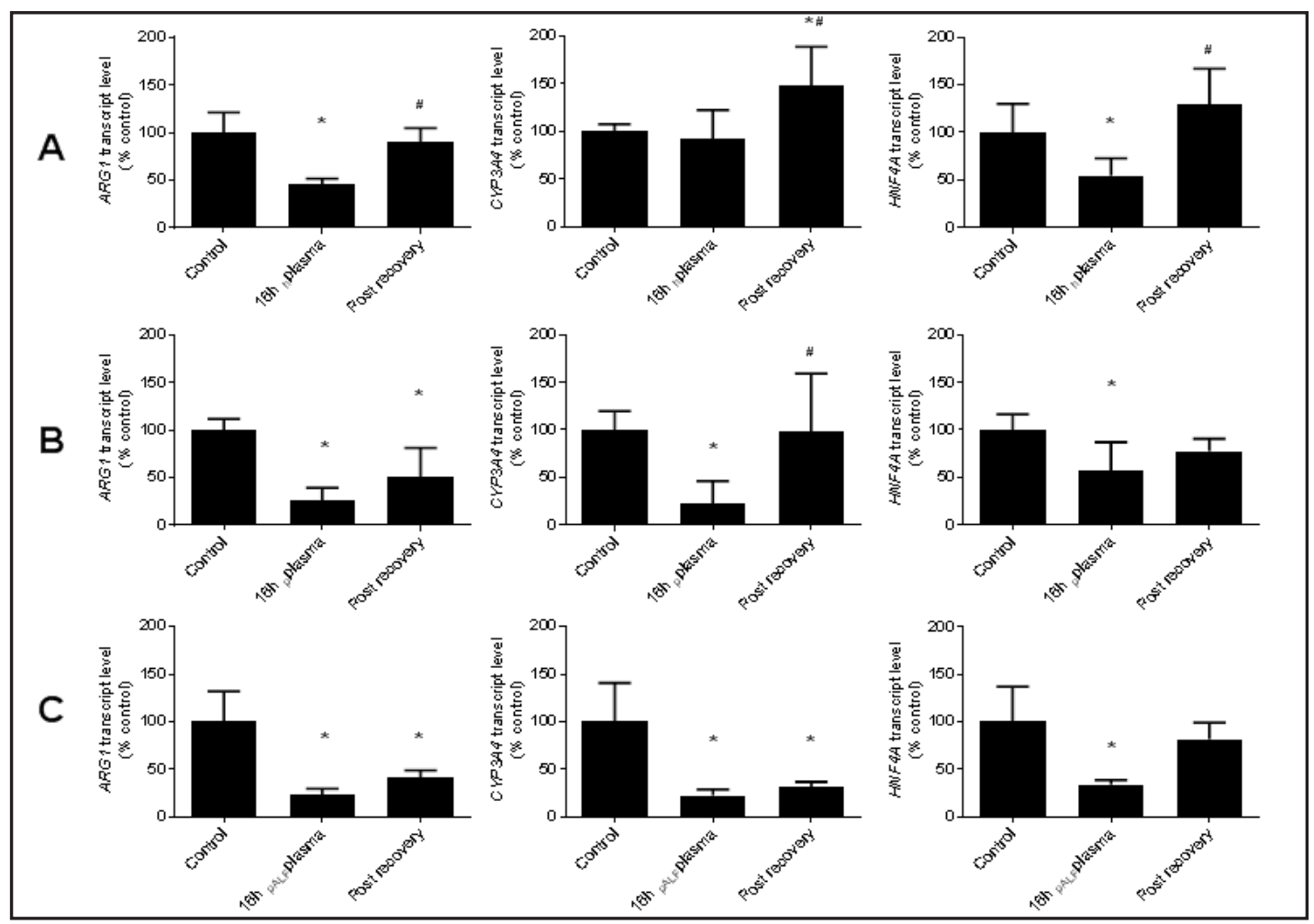

Fig. 5. Effect of ${ }_{\mathrm{h}}$ plasma, ${ }_{\mathrm{p}}$ plasma and ${ }_{\mathrm{pALF}}$ plasma on BAL-cultured HepaRG cells and recovery procedure. Transcript levels of hepatic genes ARG1, CYP3A4 and HNF4A in BAL-cultured HepaRG cells before exposure (control), directly after 16 hours of plasma-exposure and after 24 hours recovery in culture medium, expressed as a \% of the transcript levels in non-exposed BAL-cultured HepaRG cells. Three types of plasma were applied: A) ${ }_{\mathrm{h}}$ plasma, B) ${ }_{\mathrm{p}}$ plasma, $\mathrm{C}{ }_{\mathrm{pALF}}$ plasma. ${ }^{*}=P<0.05$ versus control. $\#=P<0.05$ versus post-plasma. 
Porcine ALF plasma
induces additional
mitochondrial damage
compared to healthy
plasma

Since hepatocytes are dependent on mitochondrial activity for their high energy demanding functions, and microarray analysis indicated a shift in energy metabolism from oxidative phosphorylation towards glycolysis upon plasma exposure (Fig. 3B), we assessed mitochondrial abundance, membrane potential and mitochondrial gene transcript levels on the plasma and ${ }_{\mathrm{pALF}}$ plasmaexposed BALs.

After exposure to pALF plasma, transcript levels of the mitochondrially encoded genes MT-CYTB and MT-ND5 decreased by 3.2and 3.6-fold respectively, but after recovery they increased 1.9- and 1.7-fold respectively compared to control BALs, which were not exposed to any plasma (Fig. 6A-B). BALs exposed to plasma showed no change in transcript levels, suggesting an additional mitotoxic effect of the ALF plasma. This was confirmed by JC1-staining on samples of BAL matrix after recovery (Fig. 6C), which indicated a significant loss of mitochondrial membrane potential for ${ }_{\text {pALF }}$ plasma-exposed BALs, but not plasma-exposed BALs. However, the ratios between DNA of the mitochondrial genes MT-CO and MT-ND1 and the nuclear gene CEBPA, were unaffected in both groups (Fig. 6D), indicating that the abundance of mitochondria had not changed.

These results indicate that ${ }_{\text {pALF }}$ plasma, but not ${ }_{\mathrm{p}}$ plasma, impairs mitochondrial gene transcription, which is reversible after $16 \mathrm{~h}$ plasma exposure, and that also mitochondrial functionality is negatively affected by ${ }_{\text {pALF }}$ plasma.

\section{Discussion}

It is of paramount importance for BALs and other cell-based therapies that the applied cell source is resistant to human blood plasma. In order to improve this resistance it is vital to unravel the underlying mechanism. In this study we showed that ${ }_{\mathrm{h}}$ plasma did not affect viability after 16 hours of exposure, but had a dose-dependent detrimental effect on the hepatic functionality and differentiation grade of HepaRG-derived hepatocytes, in close association with NF- $\mathrm{KB}$ signaling. Cells were partly protected from this basic plasma-induced damage when cultured in BALs, and partial restoration of hepatic gene transcription could be achieved by a 24-hour regeneration period on standard culture medium after plasma exposure. In addition, we found that toxicity can be attributed to an albumin-bound, and heat-resistant plasma fraction that is, at least partly, hydrophobic. In $_{h}$ plasma that was filtered 
or albumin depleted, CYP3A4 transcript levels were found to be increased, which is likely to reflect preservation of the detoxification system's capability to be induced by exposure to ligands.

The 3D configuration and/or medium perfusion contribute in a large part to the increased plasma-resistance observed in BALs compared to monolayer cultures. This is in accordance with a previous report that primary rat hepatocytes are protected from plasma-induced toxicity by 3D-culturing [27]. The applied 3D culture platform provides a flexible culture surface, which may be essential for maintaining cell-cell contact. Loss of adhesion is one of the first events in plasma-induced toxicity, and anchorage proteins, such as E-cadherin and the beta1-integrin receptor are known to protect against hepatocyte dedifferentiation and apoptosis [28-31]. In addition, matrix rigidity is known to limit hepatocyte differentiation, in part through transcription factor HNF4A [32].

Plasma was more toxic compared to ${ }_{\mathrm{h}}$ plasma, and ${ }_{\mathrm{pALF}}$ plasma exerted additional mitochondrial toxicity, underlining that ALF plasma is a hostile environment for both the patient and cell-based medicinal therapies. Due to the scarcity of human ALF plasma there was no opportunity to assess the effect of plasma from ALF patients. In this study we used a model of paracetamol-induced liver failure which is commonly associated with mitotoxicity and disruption of cell tight junctions [33], although also ALF-plasma of other origins has been reported to impair mitochondrial activity to varying degrees [31, 34]. However, the detrimental effects of ALF-plasma are likely to vary between etiologies, patients and clinical status, implicating the necessity of close monitoring of biocomponent functionality during therapy. This way, the BAL can be replaced after reaching critical plasma-induced toxicity of its biocomponent. Yet, it would be advantageous to inhibit at least the basic plasma-induced toxicity caused by toxic components already present in healthy plasma, which likely varies to lesser extent between individuals.

Hepatic gene transcript levels started to decline as early as four hours after ${ }_{\mathrm{h}}$ plasma exposure, preceded by upregulation of pro-inflammatory genes within one hour, which was associated with upregulation of the pro-inflammatory NF- $\kappa B$ signaling-pathway. To further elucidate the role of NF- $\kappa \mathrm{B}$, we treated HepaRG cells with NF- $\kappa \mathrm{B}$-inhibitors sulfasalazine and JSH-23. Unfortunately, these compounds did not effectively inhibit NF- $\mathrm{KB}$ activation when applied in maximum non-toxic dose (data not shown). A possible explanation is that the chemical inhibitors are metabolized by the cells. Therefore, the functionally participation of $\mathrm{NF}-\mathrm{\kappa B}$ in mediating the upregulation of pro-inflammatory genes could not be established.

Yet, NF-кB -associated pro-inflammatory cytokines, such as IL-6, are known to have a dedifferentiating effect on primary hepatocytes $[35,36]$. Especially for drug-detoxification enzymes, it is well documented that pro-inflammatory cytokines, and particularly IL-6, downregulate functionality through inhibition of transcription $[37,38]$. These mechanisms were confirmed in HepaRG cells by others: pro-inflammatory cytokines suppressed transcription of phase 1 and phase 2 detoxification enzymes and drug transporters, as well as CYP450-enzyme activities [39].

There are several isoforms of NF- $\kappa \mathrm{B}$, with partially overlapping signaling cascades, exerting a multitude of effects. Pan-inhibition of NF- $\mathrm{KB}$ is known to induce and exacerbate hepatocyte apoptosis $[40,41]$, therefore this is not an attractive strategy to counter plasma toxicity. It has been described that the RELA/p65 isoform leads to inflammatory cytokine production, posing a more specific target. However, genetic disruption of this pathway is also known to sensitize hepatocytes to apoptosis $[42,43]$.

Ideally, plasma toxicity should be inhibited by targeting more upstream processes. We have established that the detrimental compound(s) reside(s) in a ${ }_{\mathrm{h}}$ plasma fraction that is albumin bound, non-polar and heat resistant. NF- $\mathrm{KB}$ can be activated through extracellular receptor signaling, such as Toll-Like Receptors, which are classically stimulated by bacterial and viral particles, mitochondrial reactive oxygen species (ROS)-production and hypoxia $[44,45]$. We found that antioxidant treatment of HepaRG cells with N-acetyl cysteine did not decrease the detrimental plasma effects (data not shown), indicating that ROS is unlikely to be a main contributor to ${ }_{h}$ plasma toxicity [45]. NF- $\kappa B$ activation through hypoxia-induced 
pathways might be an option, as our microarray data showed that oxidative phosphorylation is severely affected. This does not necessarily imply a direct association with hypoxia; culturing the HepaRG cells and exposing them to plasma under hyperoxic conditions did not yield any protection against plasma toxicity.

In case of direct receptor stimulation, there is the possibility to inhibit plasma toxicity by molecular interference. Plasmapheresis filters with high affinity for Toll-like receptor 2 ligands ameliorated clinical symptoms of ALF in pigs with paracetamol overdose [46]. However, for specific targeting, further studies into the nature of the toxic component in plasma are required. Specific detoxification modules could easily be integrated into the plasmapheresis set-up, potentially protecting both the biocomponent and the native liver. A hybrid system, combining BAL-treatment with albumin dialysis may reduce damage of plasma to the biocomponent. One study, performed with human ALF plasma, addressed this option and showed, however, that albumin dialysis of the plasma did not reduce its proapoptotic effect on hepatocytes [31]. This may indicate that the toxic fraction has a very high affinity for albumin, since dialysis only removes the unbound fraction, and compounds with high binding affinities are therefore not removed efficiently.

Alternatively, the basic plasma-induced toxicity can be prevented by filtration for molecular size; plasma passed through a molecular weight cut-off filter of $100 \mathrm{kDa}$ was no longer toxic. A disadvantage of small-pore plasma filters is that the mass-transfer of compounds during BAL therapy may be limited, which will decrease its efficacy. Others have previously studied the optimal plasmapheresis filter pore size, and concluded that a highconvection $400 \mathrm{kDa}$ cut-off membrane offers the optimal balance between protection of the biocomponent and mass-transfer of toxins [47].

As an alternative strategy, the negative effects of basic plasma on hepatocytes can also be reversed by limiting the exposure-time to plasma so that damage to the biocomponent is still reversible. Our data show that gene transcription of HepaRG-BAL cultures can be restored after 16 hours of ${ }_{h}$ plasma exposure by recirculating culture medium through the device, indicating that alternated treatment- and restoration runs are also a viable strategy to increase the life-span of BALs, as proposed by others previously [7].

In conclusion, ${ }_{\mathrm{h}}$ plasma has a detrimental effect on differentiated HepaRG cultures, which is associated with early NF- $\mathrm{BB}$ activation. Strategies to extend functional time in BAL set-up include intermitted exposure- and recovery runs, the use of small-pore size and/or cytokine scavenging plasmapheresis filters and direct inhibition of involved pathways, possibly such as NF- $\mathrm{BB}$ signaling. ALF plasma may elicit additional toxic effects, which may vary between patients. Therefore, close monitoring of the functionality will be required for optimal BAL therapy of ALF patients.

\section{Abbreviations}

ALF (Acute liver failure); AMC (Academic Medical Center); ARG1 (Arginase1); BAL (Bioartificial liver); CYP3A4 (Cytochrome P450 3A4); DMSO (Dimethyl sulfoxide); HNF4A (Hepatic Nuclear Factor 4 Alpha); ${ }_{\mathrm{h}}$ plasma (Healthy human plasma); IL-6 (Interleukin 6); $I L-8$ (Interleukin 8); NF- $\kappa$ B (Nuclear factor kappa-light-chain-enhancer of activated B cells); ${ }_{\mathrm{pAL}}$ plasma (Porcine ALF plasma); ${ }_{\mathrm{p}}$ plasma (Healthy porcine plasma); ROS (Reactive oxygen species); TLR (Toll-like receptor);

\section{Acknowledgements}

The authors thank Sander Meissner and Vanessa Muncan for performing the microarray experiment and Erik Hendriks for assisting with the experiments and analyses. The research leading to these results has received funding from the European Community's Seventh Framework Programme FP7/2007-2013 under grant agreement 304914. 


\section{Cellular Physiology Cell Physiol Biochem 2018;48:2189-2204 \begin{tabular}{l|l} 
and Biochemistry Published 10.1159/000492560 & $\begin{array}{l}\text { (c) } 2018 \text { The Author(s). Published by S. Karger AG, Basel } \\
\text { www.karger.com/cpb }\end{array}$
\end{tabular}}

van Wenum et al: Healthy- and Acute Liver Failure -Plasma Induced Toxicity

\section{Disclosure Statement}

Robert Chamuleau is Chief Scientific Officer of the university spin-off company HepArt Medical Devices B.V that holds the exclusive license to the AMC-Bioartificial liver. Ruurdtje Hoekstra was previously employed part-time by Hep-Art Medical Devices B.V. The authors have no other relevant affiliations or financial involvement with any organization or entity with a financial interest in or financial conflict with the subject matter or materials discussed in the manuscript apart from those disclosed.

\section{References}

1 Bernal W, Wendon J: Acute liver failure. N Engl J Med 2013;26:2525-2534.

2 Glorioso JM, Mao SA, Rodysill B, Mounajjed T, Kremers WK, Elgilani F, Hickey RD, Haugaa H, Rose CF, Amiot B, Nyberg SL: Pivotal preclinical trial of the spheroid reservoir bioartificial liver. J Hepatol 2015;2:388-398.

3 Shi XL, Gao Y, Yan Y, Ma H, Sun L, Huang P, Ni X, Zhang L, Zhao X, Ren H, Hu D, Zhou Y, Tian F, Ji Y, Cheng $X$, Pan G, Ding YT, Hui L: Improved survival of porcine acute liver failure by a bioartificial liver device implanted with induced human functional hepatocytes. Cell Res 2016;2:206-216.

-4 Nibourg GA, Chamuleau RA, van der Hoeven TV, Maas MA, Ruiter AF, Lamers WH, Oude Elferink RP, van Gulik TM, Hoekstra R: Liver progenitor cell line HepaRG differentiated in a bioartificial liver effectively supplies liver support to rats with acute liver failure. PLoS One 2012;6:e38778.

5 Nagamoto Y, Takayama K, Ohashi K, Okamoto R, Sakurai F, Tachibana M, Kawabata K, Mizuguchi H: Transplantation of a human iPSC-derived hepatocyte sheet increases survival in mice with acute liver failure. J Hepatol 2016;5:1068-1075.

6 Dhawan A: Clinical human hepatocyte transplantation: Current status and challenges. Liver Transpl 2015;S39-44.

7 Stefanovich P, Matthew HW, Toner M, Tompkins RG, Yarmush ML: Extracorporeal plasma perfusion of cultured hepatocytes: effect of intermittent perfusion on hepatocyte function and morphology. J Surg Res 1996;1:57-63.

8 Matthew HW, Sternberg J, Stefanovich P, Morgan JR, Toner M, Tompkins RG, Yarmush ML: Effects of plasma exposure on cultured hepatocytes: Implications for bioartificial liver support. Biotechnol Bioeng 1996;1:100-111.

- Hughes RD, Cochrane AM, Thomson AD, Murray-Lyon IM, Williams R: The cytotoxicity of plasma from patients with acute hepatic failure to isolated rabbit hepatocytes. Br J Exp Pathol 1976;3:348-353.

10 Catapano G, De Bartolo L, Lombardi CP, Drioli E: The effect of catabolite concentration on the viability and functions of isolated rat hepatocytes. Int J Artif Organs 1996;4:245-250.

11 Pagani R, Portoles MT, Diaz-Laviada I, Municio AM: Morphological damage induced by Escherichia coli lipopolysaccharide in cultured hepatocytes: localization and binding properties. Br J Exp Pathol 1988;4:537-549.

12 Flendrig LM, la Soe JW, Jorning GG, Steenbeek A, Karlsen OT, Bovee WM, Ladiges NC, te Velde AA, Chamuleau RA: In vitro evaluation of a novel bioreactor based on an integral oxygenator and a spirally wound nonwoven polyester matrix for hepatocyte culture as small aggregates. J Hepatol 1997;6:13791392.

13 van Wenum M, Adam AA, Hakvoort TB, Hendriks EJ, Shevchenko V, van Gulik TM, Chamuleau RA, Hoekstra R: Selecting Cells for Bioartificial Liver Devices and the Importance of a 3D Culture Environment: A Functional Comparison between the HepaRG and C3A Cell Lines. Int J Biol Sci 2016;8:964-978.

14 Gripon P, Rumin S, Urban S, Le Seyec J, Glaise D, Cannie I, Guyomard C, Lucas J, Trepo C, Guguen-Guillouzo C: Infection of a human hepatoma cell line by hepatitis B virus. Proc Natl Acad Sci U S A 2002;24:1565515660.

15 Hoekstra R, Nibourg GA, van der Hoeven TV, Ackermans MT, Hakvoort TB, van Gulik TM, Lamers WH, Elferink RP, Chamuleau RA: The HepaRG cell line is suitable for bioartificial liver application. Int J Biochem Cell Biol 2011;10:1483-1489. 


\section{Cellular Physiology Cell Physiol Biochem 2018;48:2189-2204

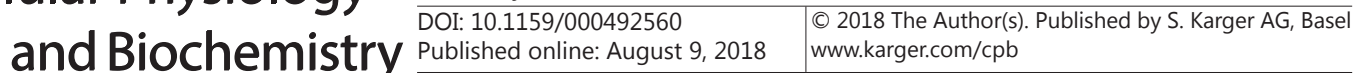

16 Hoekstra R, Nibourg GA, van der Hoeven TV, Ackermans MT, Hakvoort TB, van Gulik TM, Oude Elferink RP, Chamuleau RA: The effect of rat acute-liver-failure plasma on HepaRG cells. Int J Artif Organs 2012;11:1006-1014.

-17 Nibourg GA, Hoekstra R, van der Hoeven TV, Ackermans MT, Hakvoort TB, van Gulik TM, Chamuleau RA: Effects of acute-liver-failure-plasma exposure on hepatic functionality of HepaRG-AMC-bioartificial liver. Liver Int 2013;4:516-524.

18 Nibourg GA, Hoekstra R, van der Hoeven TV, Ackermans MT, Hakvoort TB, van Gulik TM, Chamuleau RA: Increased hepatic functionality of the human hepatoma cell line HepaRG cultured in the AMC bioreactor. Int J Biochem Cell Biol 2013;8:1860-1868.

-19 van Wenum M, Treskes P, Tang CY, Coppens EY, Jansen K, Hendriks EJ, Camus S, van Gulik TM, Chamuleau RA, Hoekstra R: Scaling-up of a HepaRG progenitor cell based bioartificial liver; optimization for clinical application and transport. Biofabrication 2017;9:035001.

20 Poyck PP, Hoekstra R, Chhatta A, Bloemendaal LT, van Wijk AC, Galavotti D, van Gulik TM, Chamuleau RA: Time-related analysis of metabolic liver functions, cellular morphology, and gene expression of hepatocytes cultured in the bioartificial liver of the Academic Medical Center in Amsterdam (AMC-BAL). Tissue Eng 2007;6:1235-1246.

21 Newsome PN, Henderson NC, Nelson LJ, Dabos C, Filippi C, Bellamy C, Howie F, Clutton RE, King T, Lee A, Hayes PC, Plevris JN: Development of an invasively monitored porcine model of acetaminophen-induced acute liver failure. BMC Gastroenterol 2010;34.

22 Bligh EG, Dyer WJ: A rapid method of total lipid extraction and purification. Can J Biochem Physiol 1959;8:911-917.

23 Hoekstra R, Deurholt T, Poyck PP, ten Bloemendaal L, Chhatta AA: Increased reproducibility of quantitative reverse transcriptase-PCR. Anal Biochem 2005;2:376-379.

-24 Su AI, Cooke MP, Ching KA, Hakak Y, Walker JR, Wiltshire T, Orth AP, Vega RG, Sapinoso LM, Moqrich A, Patapoutian A, Hampton GM, Schultz PG, Hogenesch JB: Large-scale analysis of the human and mouse transcriptomes. Proc Natl Acad Sci U S A 2002;7:4465-4470.

25 Hsiao LL, Dangond F, Yoshida T, Hong R, Jensen RV, Misra J, Dillon W, Lee KF, Clark KE, Haverty P, Weng Z, Mutter GL, Frosch MP, MacDonald ME, Milford EL, Crum CP, Bueno R, Pratt RE, Mahadevappa M, Warrington JA, Stephanopoulos G, Stephanopoulos G, Gullans SR: A compendium of gene expression in normal human tissues. Physiol Genomics 2001;2:97-104.

26 Garner DL, Thomas CA: Organelle-specific probe JC-1 identifies membrane potential differences in the mitochondrial function of bovine sperm. Mol Reprod Dev 1999;2:222-229.

27 Nagaki M, Naito T, Ohnishi H, Akaike T, Muto Y, Moriwaki H: Effects of plasma from patients with fulminant hepatic failure on function of primary rat hepatocytes in three-dimensional culture. Liver Int 2005;5:10101017.

28 Luebke-Wheeler JL, Nedredal G, Yee L, Amiot BP, Nyberg SL: E-cadherin protects primary hepatocyte spheroids from cell death by a caspase-independent mechanism. Cell Transplant 2009;12:1281-1287.

29 Meyer C, Dzieran J, Liu Y, Schindler F, Munker S, Muller A, Coulouarn C, Dooley S: Distinct dedifferentiation processes affect caveolin-1 expression in hepatocytes. Cell Commun Signal 2013;1:6.

-30 Newsome PN, Tsiaoussis J, Masson S, Buttery R, Livingston C, Ansell I, Ross JA, Sethi T, Hayes PC, Plevris JN: Serum from patients with fulminant hepatic failure causes hepatocyte detachment and apoptosis by a beta(1)-integrin pathway. Hepatology 2004;3:636-645.

-31 Saich R, Selden C, Rees M, Hodgson H: Characterization of pro-apoptotic effect of liver failure plasma on primary human hepatocytes and its modulation by molecular adsorbent recirculation system therapy. Artif Organs 2007;9:732-742.

32 Desai SS, Tung JC, Zhou VX, Grenert JP, Malato Y, Rezvani M, Espanol-Suner R, Willenbring H, Weaver VM, Chang TT: Physiological ranges of matrix rigidity modulate primary mouse hepatocyte function in part through hepatocyte nuclear factor 4 alpha. Hepatology 2016;1:261-275.

-33 Gamal W, Treskes P, Samuel K, Sullivan GJ, Siller R, Srsen V, Morgan K, Bryans A, Kozlowska A, Koulovasilopoulos A, Underwood I, Smith S, Del-Pozo J, Moss S, Thompson AI, Henderson NC, Hayes PC, Plevris JN, Bagnaninchi PO, Nelson LJ: Low-dose acetaminophen induces early disruption of cell-cell tight junctions in human hepatic cells and mouse liver. Sci Rep 2017;37541. 


\section{Cellular Physiology Cell Physiol Biochem 2018;48:2189-2204 \begin{tabular}{l|l|l} 
DOI: 10.1159/000492560 & and Biochemistry & $\begin{array}{l}\text { O 2018 The Author(s). Published by S. Karger AG, Basel } \\
\text { www.karger.com/cpb }\end{array}$
\end{tabular} \\ Published onlıne: August}

-34 Mitry RR, Bansal S, Hughes RD, Mieli-Vergani G, Dhawan A: In vitro effects of sera from children with acute liver failure on metabolic and synthetic activity of cryopreserved human hepatocytes. J Pediatr Gastroenterol Nutr 2009;5:604-607.

-35 Li AP, Yang Q, Vermet H, Raoust N, Klieber S, Fabre G: Evaluation of human hepatocytes under prolonged culture in a novel medium for the maintenance of hepatic differentiation: results with the model proinflammatory cytokine interleukin 6. Drug Metab Lett 2014;1:12-18.

-36 Dubois-Pot-Schneider H, Fekir K, Coulouarn C, Glaise D, Aninat C, Jarnouen K, Le Guevel R, Kubo T, Ishida S, Morel F, Corlu A: Inflammatory cytokines promote the retrodifferentiation of tumor-derived hepatocytelike cells to progenitor cells. Hepatology 2014;6:2077-2090.

-37 Aitken AE, Richardson TA, Morgan ET: Regulation of drug-metabolizing enzymes and transporters in inflammation. Annu Rev Pharmacol Toxicol 2006;123-149.

38 Jover R, Bort R, Gomez-Lechon MJ, Castell JV: Down-regulation of human CYP3A4 by the inflammatory signal interleukin-6: molecular mechanism and transcription factors involved. FASEB J 2002;13:1799-801.

-39 Klein M, Thomas M, Hofmann U, Seehofer D, Damm G, Zanger UM: A systematic comparison of the impact of inflammatory signaling on absorption, distribution, metabolism, and excretion gene expression and activity in primary human hepatocytes and HepaRG cells. Drug Metab Dispos 2015;2:273-83.

40 Iimuro Y, Nishiura T, Hellerbrand C, Behrns KE, Schoonhoven R, Grisham JW, Brenner DA: NFkappaB prevents apoptosis and liver dysfunction during liver regeneration. J Clin Invest 1998;4:802-811.

41 Bellas RE, FitzGerald MJ, Fausto N, Sonenshein GE: Inhibition of NF-kappa B activity induces apoptosis in murine hepatocytes. Am J Pathol 1997;4:891-896.

42 Geisler F, Algul H, Paxian S, Schmid RM: Genetic inactivation of RelA/p65 sensitizes adult mouse hepatocytes to TNF-induced apoptosis in vivo and in vitro. Gastroenterology 2007;7:2489-2503.

43 Ringelhan M, Schmid RM, Geisler F: The NF-kappaB subunit RelA/p65 is dispensable for successful liver regeneration after partial hepatectomy in mice. PLoS One 2012;10:e46469.

-44 Gilmore TD: Introduction to NF-kappaB: players, pathways, perspectives. Oncogene 2006;51:6680-6684.

45 Chandel NS, Trzyna WC, McClintock DS, Schumacker PT: Role of oxidants in NF-kappa B activation and TNF-alpha gene transcription induced by hypoxia and endotoxin. J Immunol 2000;2:1013-1021.

46 Lee KC, Baker LA, Stanzani G, Alibhai H, Chang YM, Jimenez Palacios C, Leckie PJ, Giordano P, Priestnall SL, Antoine DJ, Jenkins RE, Goldring CE, Park BK, Andreola F, Agarwal B, Mookerjee RP, Davies NA, Jalan R: Extracorporeal liver assist device to exchange albumin and remove endotoxin in acute liver failure: Results of a pivotal pre-clinical study. J Hepatol 2015;3:634-642.

47 Nedredal GI, Amiot BP, Nyberg P, Luebke-Wheeler J, Lillegard JB, McKenzie TJ, Nyberg SL: Optimization of mass transfer for toxin removal and immunoprotection of hepatocytes in a bioartificial liver. Biotechnol Bioeng 2009;5:995-1003. 